\title{
Relationship between pore structure and compressive strength of concrete: Experiments and statistical modeling
}

\author{
$\mathrm{J} \mathrm{BU}^{*}$ (D) and Z TIAN \\ College of Water Conservancy and Hydropower Engineering, Hohai University, Nanjing, Jiangsu 210098, \\ People's Republic of China \\ e-mail: bujingwu2008@163.com; zhtianhhu@163.com
}

MS received 9 May 2015; revised 2 July 2015; accepted 15 August 2015

\begin{abstract}
Properties of concrete are strongly dependent on its pore structure features, porosity being an important one among them. This study deals with developing an understanding of the pore structure-compressive strength relationship in concrete. Several concrete mixtures with different pore structures are proportioned and subjected to static compressive tests. The pore structure features such as porosity, pore size distribution are extracted using mercury intrusion porosimetry technique. A statistical model is developed to relate the compressive strength to relevant pore structure features.
\end{abstract}

Keywords. Pore structure; compressive strength; concrete; statistical model; mercury intrusion porosimetry (MIP).

\section{Introduction}

Concrete is a composite material of aggregates and cement pastes that fills in the spaces between aggregate particles and binds them together to form rock-like solid. Under a microscopic examination, cement paste is a non-homogeneous and anisotropic matrix composed of irregularly shaped and unevenly distributed pores attributed to the evaporation of free water and gel pore formation in the C-S-H hydrates $[1-3]$. The pore structure greatly influences the strength development of the concrete [4-7].

Substantial progress has been made in studying the effects of porosity on mechanical properties. Thus, for example, considerable documentation shows that porosity typically has a substantial effect on most mechanical properties. The compressive strength of concrete is an important parameter in civil engineering. In predicting the effect of porosity on the strength of porous materials, Hasselman \& Fulrath [8], Wagh et al [9], Liu [10], and Palchik [11] approached the problem by estimating the decrease in material available to carry the applied load. Assuming that failure occurs at a given stress level, the introduction of the first pore should cause a precipitous decrease in strength by a factor equal to the stress concentration factor of the pore. This can be partially explained by Griffith theory. According to Griffith theory, the critical stress resulting in rapid growth of crack and fracture causing failure under tension is given by: $\sigma_{t}=\sqrt{\frac{2 E T}{\pi c_{1}}}$, where $E, T$ and $c_{1}$ stand for modulus of

${ }^{*}$ For correspondence elasticity, fracture surface energy of the material and half crack length, respectively. The fracture surface energy is the energy required for creating a unit area of interface between the solid and air [12]. For porous material, the interface between the solid and air already exists at pores. So the fracture surface energy required for fracture of unit area of porous material will be lower with increase in porosity. Schiller [13] using a theoretical approach deduced an equation relating the strength of a material to the porosity. He applied this equation to experimental data on gypsum plasters and obtained a good fit for compressive and tensile strengths. A review [14] of the effect of porosity on the strength of concrete presented some of the more important empirical and theoretical equations for relating strength to porosity. The profusion of possible equation is enormous and whilst one equation is most suitable for one material a quite different equation is most suitable for a second material. Popovics [15] observes that this is true even for different types of concrete.

An increase in the porosity reduces the strength of concrete, but the magnitude of this effect depends greatly on pore size, shape, and distribution [16-18]. Some empirical and semi-empirical equations have been used with limited success to describe this phenomenon [5, 7]. Pecqueux et al [19] suggested that the effect of porosity on strength is governed by the effect of pore phase on the failure criterion on the Griffith flaws. It was hypothesized that strength is a function of pore size relative to flaw size and that stress concentrations affect strength only for a pore size of the order of or greater than the flaw size. With respect to mechanical strength, Tang [20] developed a relationship between 
pore size distribution and strength for mortar by using mercury intrusion porosimetry (MIP) data. In this model, pores are divided into different size groups and the fracture process is simulated through a computer model. The material is assumed to fracture progressively with applied stress, starting from the fraction containing the largest pores to that containing the smallest pores. The stress it withstands at the final stage of fracture is assumed as the strength of the material. However the problem with this procedure is that the strength is calculated using a computer by a numerical method needing 12 iteration steps and not by a simplified analytical method.

The purpose of this paper is to define, both theoretically and experimentally, the connection between strength and pore structure of hardened concrete. In the present work a specific model, that due to Ryshkewitch [21] has been modified to apply to the case of concrete, and calculations have made to see how this model compares with the experimental results.

\section{Experimental investigations}

\subsection{Concrete samples}

Considering the practical limits of water-cement ratios for workable concrete without water-reducing cement, five concrete mixes were designed so as to ensure adequate variation in pore structure of concrete. Ordinary Portland cement (OPC) confirming to the requirement of ASTM Type-I was used in the investigation. Distilled water prepared in the laboratory was used for the purpose of preparation of the concretes. The aggregate used were a $10-\mathrm{mm}$ nominal maximum size crushed granite and natural river sand. The mix proportions and curing ages of concrete samples are listed in table 1 . The mixing was done on a paint shaker. The

Table 1. Mix proportions and curing age of concrete samples.

\begin{tabular}{lcc}
\hline Sample no. & C:S:A:w/c & Age (days) \\
\hline A-14 & 1:1.1:2.7:0.35 & 14 \\
A-28 & & 28 \\
A-180 & & 180 \\
B-14 & $1: 1.3: 3.2: 0.4$ & 14 \\
B-28 & & 28 \\
B-180 & & 180 \\
C-14 & $1: 1.5: 3.6: 0.45$ & 14 \\
C-28 & & 28 \\
C-180 & & 280 \\
D-14 & $1: 1.8: 3.9: 0.5$ & 14 \\
D-28 & & 28 \\
D-180 & & 180 \\
E-14 & $1: 2.2: 4.2: 0.55$ & 14 \\
E-28 & & 28 \\
E-180 & & 180 \\
\hline N & &
\end{tabular}

Note: C-cement, S-sand, A-aggregate. concrete samples were cast in cubic steel molds $(10 \mathrm{~cm})$ for pore structure and compressive strength testing. After storage in a fog room for one day, the sample was demolded and put into lime-saturated water at room temperature until the test date. The range of hydration ages was from 1 to 180 days. At the predetermined ages, the specimens were taken out of the lime water, and broken into smaller pieces for intrusion. They were then dried in an oven at $105^{\circ} \mathrm{C}$ for at least $24 \mathrm{~h}$.

\subsection{Mercury intrusion porosimetry (MIP) test}

Testing was performed with a Quantachrome Autoscan 60 mercury intrusion porosimeter with a maximum intrusion pressure of $415 \mathrm{MPa}(60,000 \mathrm{psi})$. The contact angle was assumed to be $140^{\circ}$. This assumption was based on previous tests [22]. The sample cell with a capacity of $0.5 \mathrm{~cm}^{3}$, which corresponds to a maximum mass of approximately $1.8 \mathrm{~g}$, was used throughout. All the tests, except where otherwise specified, were performed on an automatic scanning rate of pressurization with each test lasting approximately $25 \mathrm{~min}$. Figure 1 compares the cumulative mercury intrusion curve as a function of pore diameter for the concrete at three ages between 14 and 180 days.

\subsection{Compression test}

The compressive strength of the cube specimens of concrete was tested with a Denison compression machine at the loading rate of $0.2-0.4 \mathrm{~N} / \mathrm{mm}^{2} / \mathrm{s}$.

\section{Model for relating pore structure to strength of concrete}

The hypothesis in this research is that compressive strength is related to the distribution of pore size determined by MIP curve. This hypothesis can be expressed in a mathematical form as follows:

$$
\sigma=\sigma_{0} \times f(V),
$$

where $f$ is a function of $V$ which is a pore size distribution from MIP, $\sigma_{0}$ is the compressive strength of the fully dense material (namely, the porosity is zero). To formulate $f(V)$, $V$ must be expressed in a mathematical equation. Since the MIP curve behaves similar to Fermic-Dirac function [23], it can be expressed as follows:

$$
V=V_{0} \frac{1}{1+\exp \left(\frac{d-d_{0}}{r}\right)},
$$

where $V(d)$ is the cumulative pore volume at a pore diameter " $d$ ", $V_{0}$ is the total pore volume, $d$ is pore diameter in $\log$ scale, $d_{0}$ is the pore diameter at the point of $50 \%$ intrusion, and $r$ is a constant describing the curvature. Usually, 

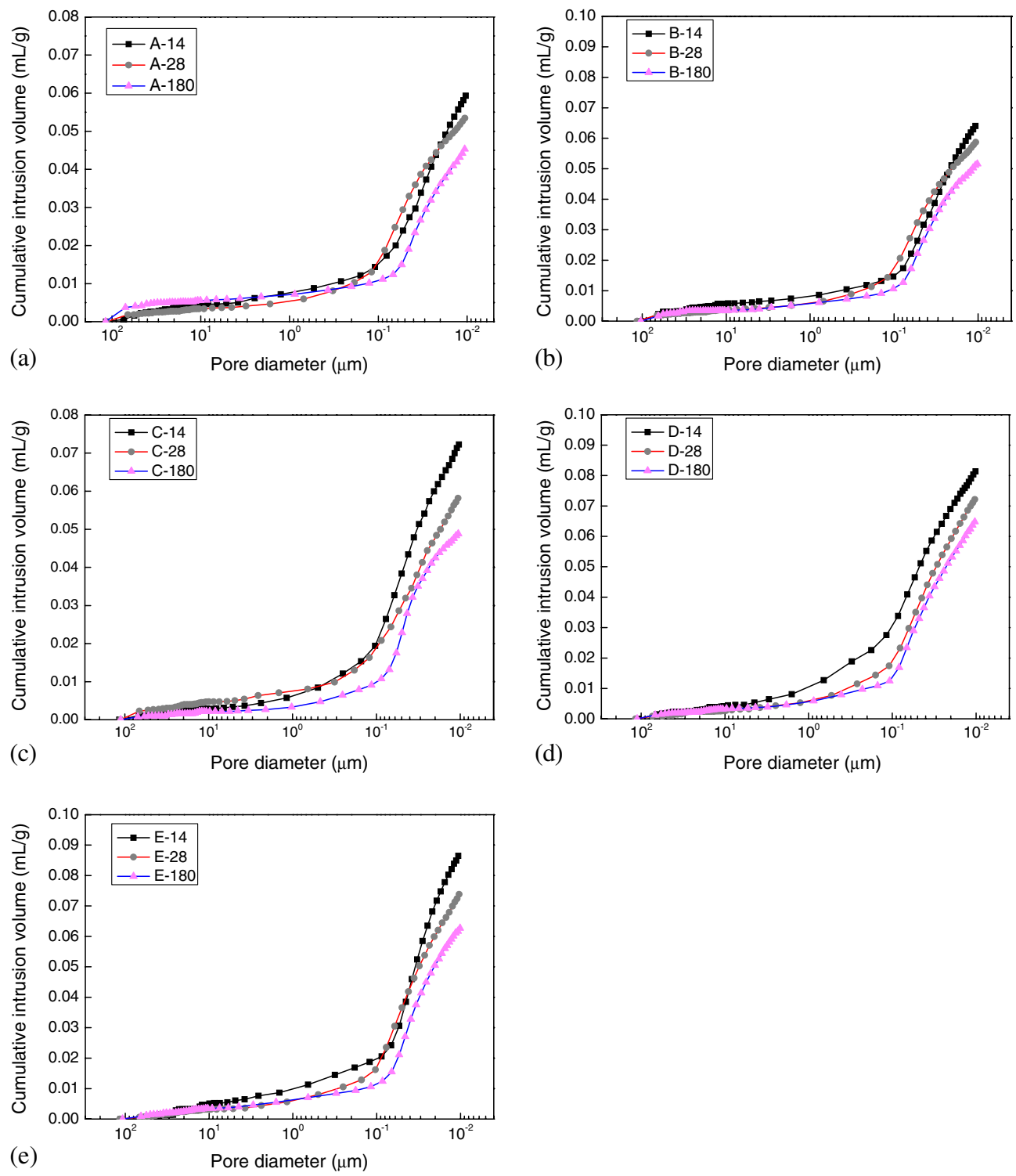

Figure 1. Cumulative volume of intrude mercury vs. pore diameter for concrete (a) mix A, (b) mix B, (c) mix C, (d) mix D, and (e) $\operatorname{mix} \mathrm{E}$.

the MIP curve has a smaller curvature when $d>d_{0}$. To accommodate this fact, $V(d)$ is expressed as follows:

$$
\begin{aligned}
& \text { if } d>d_{0}, V=V_{0} \frac{1}{1+\exp \left(\frac{d-d_{0}}{r_{1}}\right)} \\
& \text { if } d<d_{0}, V=V_{0} \frac{1}{1+\exp \left(\frac{d-d_{0}}{r_{2}}\right)} .
\end{aligned}
$$

With these terms of $V_{0}, d_{0}, r_{1}$ and $r_{2}$, the MIP curve can be characterized. In relation to these parameters, Eq. (1) can be expressed as follows:

$$
\sigma=\sigma_{0} \times f\left(V_{0}, d_{0}, r_{1}, r_{2}\right)
$$

Ryshkewitch [21] suggested the following equation for porous sintered alumina and zirconia:

$$
\sigma=\sigma_{0} \exp (-\beta p)
$$

where $p$ is the porosity, and $\beta$ is empirical constant.

With respect to multiple linear equation, the following equation is postulated:

$$
\sigma=\sigma_{0} \exp \left(b_{0}+b_{1} V_{0}+b_{2} d_{0}+b_{3} r_{1}+b_{4} r_{2}\right),
$$

where $b_{1}, b_{2}, b_{3}$ and $b_{4}$ are coefficients for $V_{0}, d_{0}, r_{1}$ and $r_{2}$, respectively and $b_{0}$ is a constant.

For Eq. (7), $p$ and $d_{0}$ can be calculated from the MIP data. However, $r_{1}$ and $r_{2}$ in Eqs. (3) and (4) is not easily calculated from raw MIP data. Figure 2 shows the calculated 

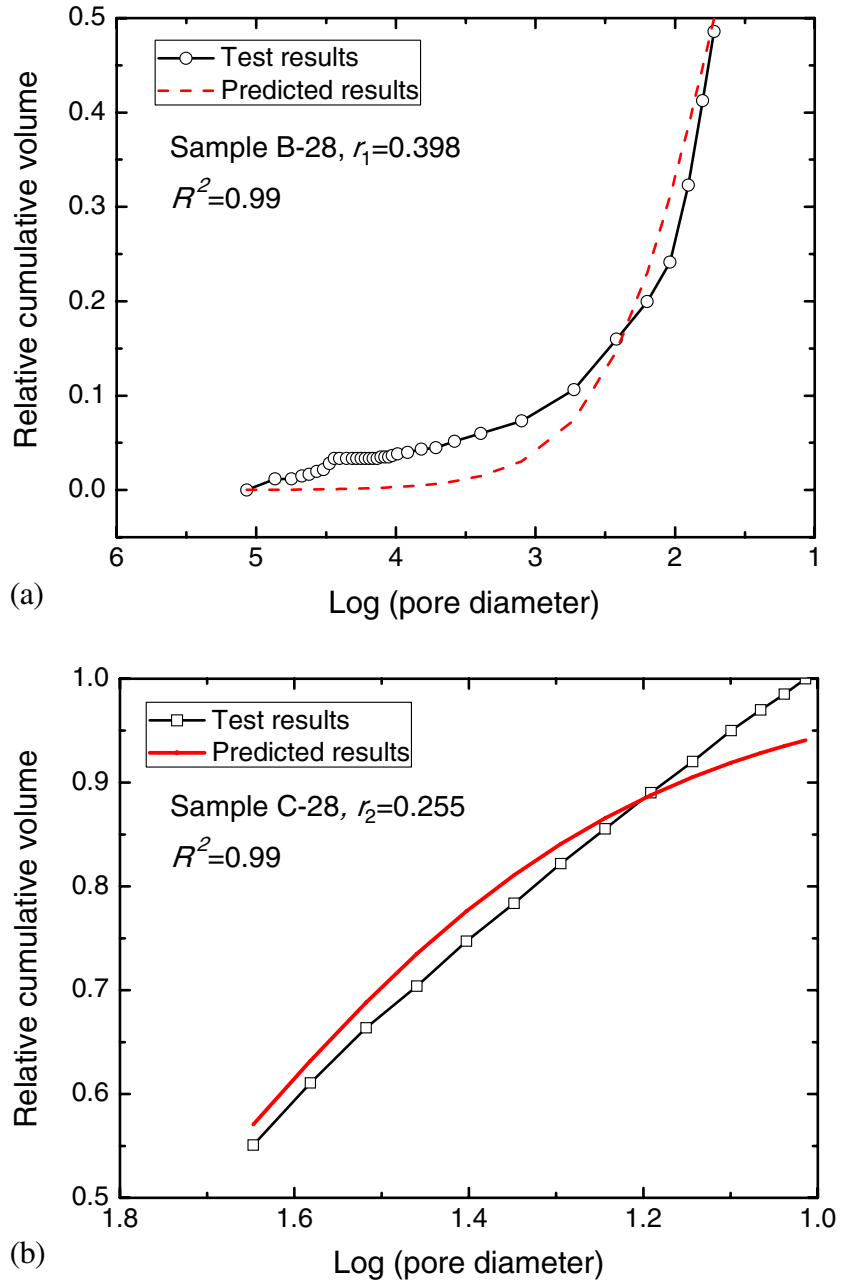

Figure 2. Actual MIP curve of sample B-28 and predicted results (a) Eq. (3), and (b) Eq. (4).

curve from curve fitting and the actual MIP curve. For the statistical analysis, computer program "1stopt" was used for computation.

As mentioned by Deo \& Neithalath [24], interaction of pores with cracks also must be considered. In this respect, the curvatures of the tip of micro-pores are important parameters. Since the front end of intruding mercury is assumed as spherical in shape, micro-pore is expected to be filled at a pressure equivalent to pore size $R_{4}$. It is assumed that the pore diameter corresponding to $90 \%$ total intrusion is $R_{4}$. Equation (4) can be a relevant function for MIP curve since its shape changes similarly with changing of $r_{2}$. Therefore, two terms, $R_{4}$ and $r_{2}^{\prime}$ are formulated with respect to the micro-pores. For this reason, the micro-pore region of the MIP curve was characterized using Eq. (4) in terms of $R_{4}$ and $r_{2}^{\prime}$ replacing $d_{0}$ and $r_{2}$, respectively, and it can be expressed as follow,

$$
F(x)=\frac{1}{1+\exp \left(\frac{R_{4}-x}{r_{2}^{\prime}}\right)} .
$$

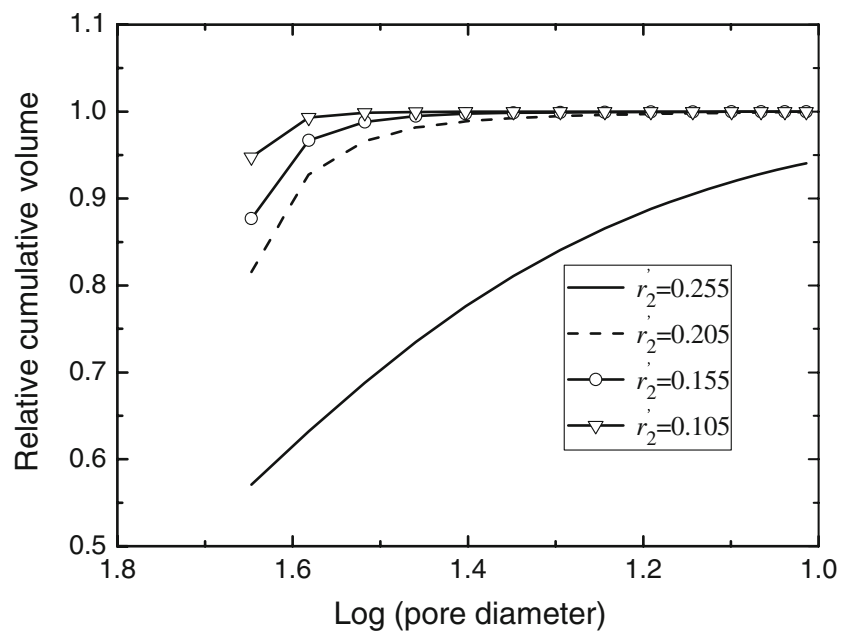

Figure 3. Plot of $F(x)$ with $d_{0}$ of 1.65 and different $r_{2}^{\prime}$ for sample B-28.

As shown in figure 3, Eq. (8) characterize the shape of MIP curve as the changing of $r_{2}^{\prime}$. The curve in micro-pore region tends to straight line with decreasing of curvature $r_{2}^{\prime}$.

Although pore shape of macro-pores cannot be characterized with MIP curves, the overall contents of macro-pores may be characterized by the slope of the MIP curve, which is a straight in the large pore region. As a new microstructural parameter characterizing the macro-pore region, Slope- $L$ is devised instead of $r_{1}$. Slope- $L$ is calculated as follows:

$$
\text { Slope- } L=\frac{V_{R_{2}^{\prime}}-V_{R_{2}}}{R_{2}-R_{2}^{\prime}},
$$

where $R_{2}$ and $R_{2}^{\prime}$ are the beginning and ending points of the straight line in macro-pore region in MIP curve.

$d_{0}$ does not represent the major threshold. Threshold diameter $R_{3}$ can be defined as the pore diameter at which largest mercury intrusion during MIP occurs, at this point the negative curvature of the cumulative mercury intrusion volume curve turns to be positive; usually this is not the same as $d_{0}$, which is the pore diameter which is equivalent to $50 \%$ of total intrusion. $R_{3}$ is another newly formulated microstructural parameter for characterizing the major threshold.

From the above background of micro-pore, macro-pore and threshold, a new set of micro-pore, macro-pore and threshold, a new set of microstructural parameters, $p$, Slope- $L$, $r_{2}^{\prime}, R_{3}, d_{0}$ and $R_{4}$ can be formulated. Their structural characteristics are summarized in table 2. Graphically these microstructural parameters are shown in figure 4 . The corresponding data for these parameters for each specimen are summarized in table 3 and used in multiple linear regressions for the strength.

Thus, a modified equation can be expressed as follows:

$$
\sigma=f\left(p, \text { Slope }-L, d_{0}, R_{3}, R_{4}, r_{2}^{\prime}\right)
$$


Table 2. Summary of parameters from MIP and their characteristic features.

\begin{tabular}{lcc}
\hline Characteristic feature & Parameter & Unit \\
\hline Total porosity & $p$ & None \\
Total amount of macro-pores & Slope-L & None \\
Threshold & $R_{3}$ & $\mu \mathrm{m}$ \\
& $d_{0}$ & $\mu \mathrm{m}$ \\
Radius of micro-pores & $R_{4}$ & $\mu \mathrm{m}$ \\
Shape of micro-pores & $r_{2}^{\prime}$ & None \\
\hline
\end{tabular}

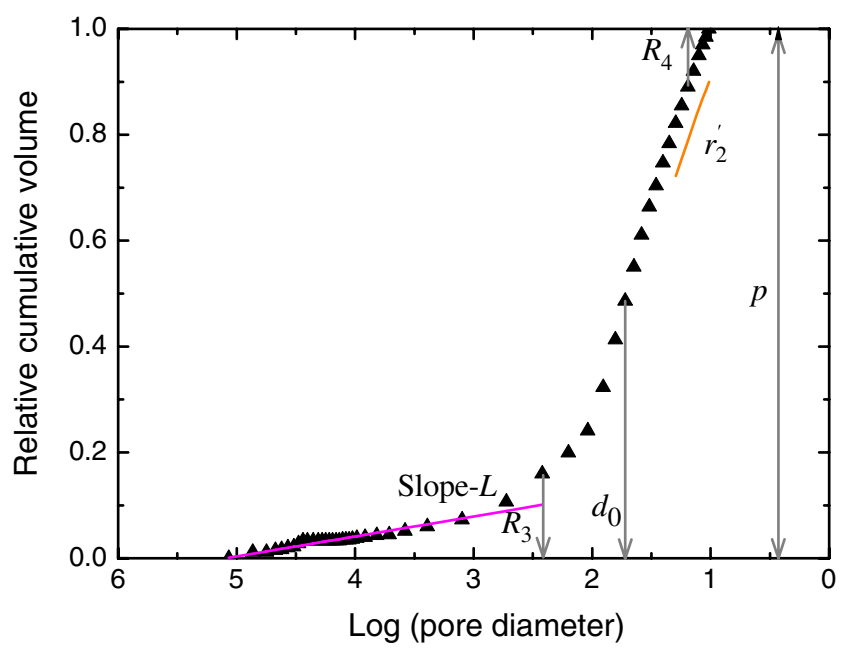

Figure 4. Microstructural parameters shown in a typical MIP curve.

The equation was tested by multiple linear regression assuming the following model equation for the relationship between strength and the microstructural parameters.

$$
\ln \left(\frac{\sigma}{\sigma_{0}}\right)=\sum_{i} b_{i} X_{i}
$$

where $X_{i}$ represents each of the microstructural parameters, $p$, Slope- $L, r_{2}^{\prime}, d_{0}, R_{3}$, and $R_{4}$. Among these parameters, three parameters, $d_{0}, R_{3}$, and $R_{4}$, have the same characteristic features representing radius of pores although $R_{4}$ represents the radius of micro-pores.

\section{Results and discussions}

\subsection{Determination of the statistical model}

As expected, a multiple non-linear regression model was found to provide the highest degree of predictive capability. However, when all the pore structure features were considered individually in a non-linear regression model, issues with multicollinearity were observed. Multicollinearity is a phenomenon in which two or more model terms are highly correlated to each other, and this correlation coefficient is greater than the correlation of these terms with the response variable [25]. While this in itself does not reduce the predictive capability of the model, it might interfere with the validity of assigning sensitivity to the individual variables. Statistics of the parameters used for regression analysis are summarized in table 3 . The correlation matrix in table 4 reveals the general relationship between the dependent variable $\ln \left(\sigma / \sigma_{0}\right)$ and independent variables and between independent variables. Among these parameters, $p$ and Slope- $L$ have high correlation coefficients greater than 0.9. This high correlation means that these two parameters should be entered in the statistical analysis. Also, among $R_{3}, R_{4}$ and $d_{0}$, which represent diameters of the pores, $d_{0}$ has a highest correlation coefficient with strength. Considering the correlation between the independent variables (microstructural parameters), high correlations are seen to exist between $p$ and Slope $-L(0.899), r_{2}^{\prime}$ and $R_{3}(0.624), r_{2}^{\prime}$ and $d_{0}(0.62)$, $R_{3}$ and $d_{0}(0.975)$. This high correlation between independent variables indicates that Eq. (11) does not need all of microstructural parameters.

Table 3. Summary of microstructural parameters with strength for statistical analysis.

\begin{tabular}{lccccccc}
\hline Sample no. & $p$ & $d_{0}$ & Slope- $L$ & $R_{3}$ & $R_{4}$ & $r_{2}^{\prime}$ & Strength $(\mathrm{MPa})$ \\
\hline A-14 & 15.27 & 1.09 & 0.0383 & 1.20 & 1.0325 & 0.198 & 41.38 \\
A-28 & 11.93 & 1.15 & 0.00367 & 1.31 & 1.0372 & 0.231 & 47.17 \\
A-180 & 10.30 & 1.09 & 0.00267 & 1.17 & 1.0301 & 0.227 & 50.33 \\
B-14 & 16.85 & 1.09 & 0.0497 & 1.19 & 1.0327 & 0.222 & 36.54 \\
B-28 & 14.82 & 1.14 & 0.00803 & 1.32 & 1.0334 & 0.255 & 42.65 \\
B-180 & 13.05 & 1.11 & 0.00419 & 1.20 & 1.0358 & 0.201 & 46.28 \\
C-14 & 18.74 & 1.13 & 0.0618 & 1.27 & 1.0349 & 0.220 & 32.56 \\
C-28 & 15.31 & 1.19 & 0.0396 & 1.32 & 1.0361 & 0.261 & 38.77 \\
C-180 & 14.30 & 1.11 & 0.0384 & 1.17 & 1.0418 & 0.197 & 41.08 \\
D-14 & 18.66 & 1.17 & 0.0802 & 1.31 & 1.0361 & 0.279 & 27.34 \\
D-28 & 15.88 & 1.12 & 0.0559 & 1.28 & 1.0325 & 0.255 & 34.49 \\
D-180 & 14.38 & 1.12 & 0.0417 & 1.21 & 1.0334 & 0.236 & 38.21 \\
E-14 & 20.97 & 1.10 & 0.0839 & 1.19 & 1.0361 & 0.173 & 24.91 \\
E-28 & 17.02 & 1.12 & 0.0699 & 1.27 & 1.0349 & 0.243 & 31.19 \\
E-180 & 15.71 & 1.09 & 0.0569 & 1.17 & 1.0339 & 0.254 & 33.78 \\
\hline
\end{tabular}


Table 4. Statistics of data set and correlation data.

\begin{tabular}{lcccccc}
\hline & $\ln \left(\sigma / \sigma_{0}\right)$ & $p$ & Slope $-L$ & $r_{2}^{\prime}$ & $R_{4}$ & $R_{3}$ \\
\hline $\ln \left(\sigma / \sigma_{0}\right)$ & 1 & -0.942 & -0.961 & 0.299 & -0.166 & -0.293 \\
$p$ & & 1 & 0.899 & -0.141 & 0.0239 & 0.356 \\
Slope- $L$ & & 1 & -0.432 & 0.117 & 0.392 \\
$r_{2}^{\prime}$ & & & 1 & -0.224 & 0.0245 \\
$R_{4}$ & & & & 1 & 0.0124 \\
$R_{3}$ & & & & & 0.101 \\
$d_{0}$ & & & & & 0.319 \\
\hline
\end{tabular}

Table 5. List of model selection criteria, $R^{2}, A I C$ and $\chi^{2}$ for combination of microstructural parameters.

\begin{tabular}{|c|c|c|c|c|c|c|c|c|c|}
\hline Case & $p$ & Slope- $L$ & $d_{0}$ & $R_{3}$ & $R_{4}$ & $r_{2}^{\prime}$ & $R^{2}$ & $|A I C|$ & $\left|\chi^{2}\right|$ \\
\hline 1 & $*$ & & & & & & 0.942 & 7.9 & 0.03 \\
\hline 2 & $*$ & $*$ & & & & & 0.964 & 0.73 & 0.023 \\
\hline 3 & $*$ & $*$ & $*$ & & $*$ & & 0.978 & 1.36 & 0.012 \\
\hline 4 & $*$ & $*$ & $*$ & $*$ & & & 0.978 & 0.59 & 0.012 \\
\hline 5 & $*$ & $*$ & $*$ & & & & 0.978 & 0.66 & 0.012 \\
\hline 6 & $*$ & $*$ & $*$ & & & $*$ & 0.98 & 0.54 & 0.011 \\
\hline 7 & $*$ & $*$ & $*$ & $*$ & & $*$ & 0.981 & 2.6 & 0.013 \\
\hline
\end{tabular}

* The corresponding pore structure parameter is selected in the statistical model.

Although the correlation table (table 4 ) indicates the significance of $p$, Slope- $L$ and $R_{3}$, the selection of microstructural parameters as the best independent variables must be completed with selection criteria such as $R^{2}$ (correlation coefficient), AIC (Aikake's Information Criterion) and $\chi^{2}$ (Chi-Square) test [26].

$R^{2}$ (correlation coefficient) reflects relationship between two variables, the higher the value is, the closer the fitting results to experimental data.

AIC (Aikake's Information Criterion) is a criterion to evaluate statistical model, it can be expressed as

$$
A I C=(2 K-2 L) / n,
$$

where $K$ is the number of parameters, $L$ is logarithmic likelihood function, and $n$ is number of observations. $K$ represents the simplification of the model, and the less $K$ demonstrated the model is more simplified. Larger $L$ indicates the model is more accurate. Therefore, AIC can test the simplification and accuracy of a model, and the less the value of AIC, the better the model is. The statistic of $\chi^{2}$ (Chi-Square) test is $\chi^{2}$ value, which is a cumulative sum of the ratio of the square of difference between experimental frequency and theoretical frequency to theoretical frequency. The larger absolute value of $\chi^{2}$ represents the more obvious difference between experimental and theoretical results. As a consequence, a model with largest $R^{2}$, least $A I C$ and $\chi^{2}$ value is the best.

These selection criteria are given in table 5. From the results of statistical analysis, the best model is Case 6 . Therefore the relationship of compressive strength with these microstructural parameters can be expressed as follows:

$\ln \left(\frac{\sigma}{\sigma_{0}}\right)=\sum_{i} b_{i} X_{i}=b_{\text {Slope }-L} \cdot$ Slope $-L+b_{p} \cdot p+b_{r_{2}^{\prime}} \cdot r_{2}^{\prime}+b_{d_{0}} \cdot d_{0}$.

In an exponential form, Eq. (13) can be expressed as

$\sigma=\sigma_{0} \exp \left(b_{p} \cdot p+b_{\text {Slope- } L} \cdot\right.$ Slope- $\left.L+b_{r_{2}^{\prime}} \cdot r_{2}^{\prime}+b_{d_{0}} \cdot d_{0}\right)$.

Inserting the $b_{i}$ from regression analysis, Eq. (14) becomes as

$\sigma=\sigma_{0} \exp \left(-0.032 p-0.244 d_{0}-4.073\right.$ Slope $\left.-L-0.317 r_{2}^{\prime}\right)$.

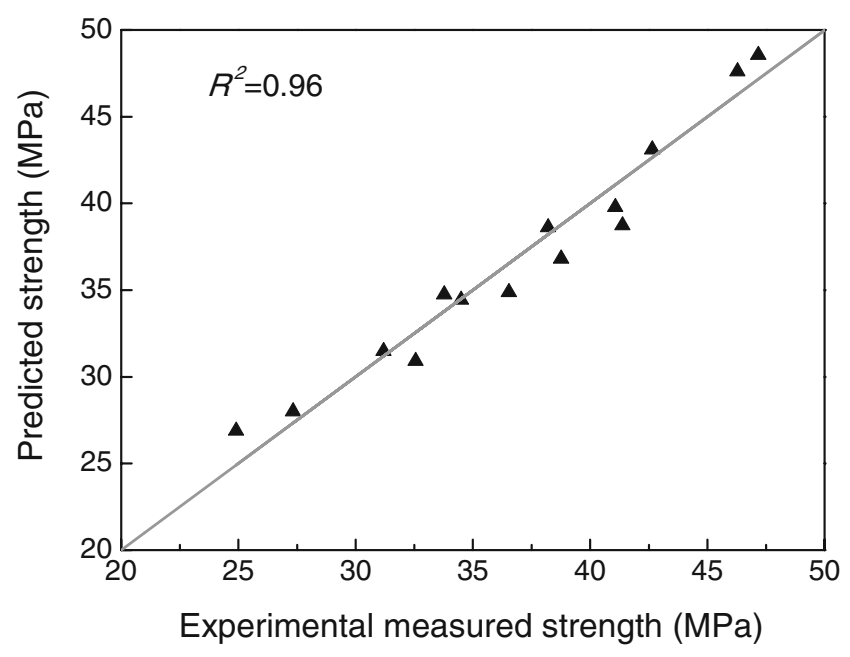

Figure 5. Predicted vs. actual strength for all concrete specimens. 


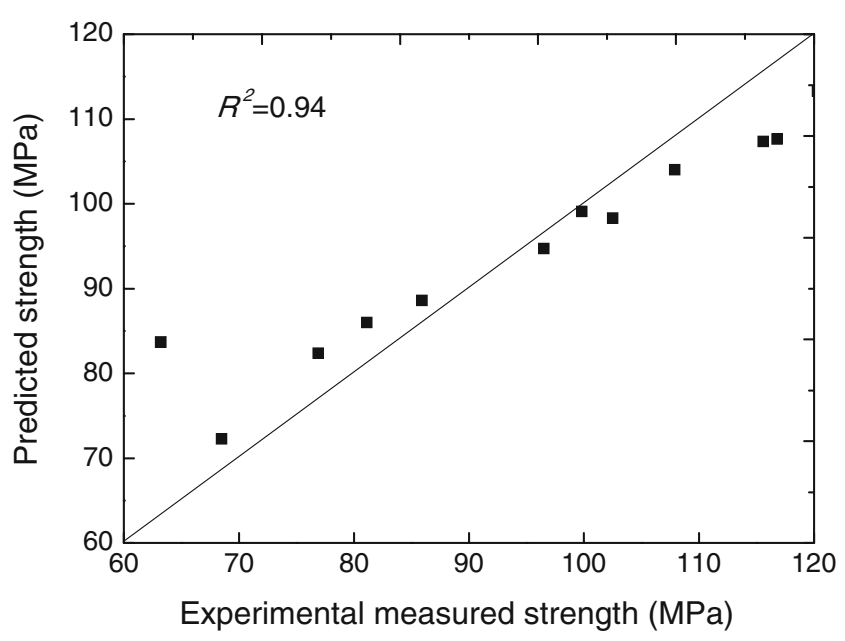

Figure 6. Predicted vs. actual strength for concrete specimens [27].

\subsection{Verification of the proposed model}

The accuracy of the model in predicting the compressive strength of concrete under the experimental conditions is shown in figure 5 where actual vs. predicted results is plotted for all mixtures. In order to be shown to be of general usefulness, the empirical equation obtained in this paper is also verified using several other concrete mixes from Poon et al [27] and the experimental vs. predicted strengths are plotted in figure 6 . The model predictions of the strength are found to be acceptable.

\section{Conclusions}

This study has focused on relating the pore structure features of concrete to the measured compressive strength. It is shown that the pore structure features other than porosity are also responsible for the compressive strength of concrete. An equation for calculating strength included pore structure parameters extracted from MIP data expressed as Eq. (15). Among these pore structure parameters, slope and curvature are characteristic features extracted from MIP curve and are related to macro-pores and micro-pores, respectively. Strength predicted using this equation is in good accuracy with experimental values.

\section{Acknowledgements}

Financial support for this research was provided by the National Natural Science Foundation of China (Grant Nos. 51279054 and 51279052).

\section{References}

[1] Bouguerra A, Amiri O, Ait-Mokhtar A and Diop M B 2002 Water sorptivity and pore structure of wood-cementitious composites. Mag. Concr. Res. 54(2): 103-112
[2] Diamond S 2004 The microstructure of cement paste and concrete - a visual primer. Cem. Concr. Compos. 26(6): 919933

[3] Stark J 2011 Recent advances in the field of cement hydration and microstructure analysis. Cem. Concr. Res. 41(5): 666678

[4] Kearsley E P and Wainwright P J 2002 The effect of porosity on the strength of foamed concrete. Cem. Concr. Res. 32(2): 233-239

[5] Kumar R and Bhattacharjee B 2003 Porosity, pore size distribution and in situ strength of concrete. Cem. Concr. Res. 33(1): 155-164

[6] Marfisi E, Burgoyne C J, Amin M H G and Hall L D 2005 The use of MRI to observe the structure of concrete. Mag. Concr. Res. 57(2): 101-109

[7] Lian C, Zhuge Y and Beecham S 2011 The relationship between porosity and strength for porous concrete. Constr. Build. Mater. 25(9): 4294-4298

[8] Hasselman D P H and Fulrath R M 1966 Proposed fracture theory of a dispersion-strengthened glass matrix. J. Am. Ceram. Soc. 49(2): 68-72

[9] Wagh A S, Singh J P and Poeppel R B 1993 Dependence of ceramic fracture properties on porosity. J. Mater. Sci. 28(11): 3589-3593

[10] Liu D-M 1997 Influence of porosity and pore size on the compressive strength of porous hydroxyapatite ceramics. Ceram. Int. 23(2): 135-139

[11] Palchik V 1999 Influence of porosity and elastic modulus on uniaxial compressive strength in soft brittle porous sandstones. Rock Mech. Rock Eng. 32(4): 303-309

[12] Luping T 1986 A study of the quantitative relationship between strength and pore size distribution of the porous materials. Cem. Concr. Res. 16(1): 87-96

[13] Schiller K K 1971 Strength of porous materials. Cem. Concr. Res. 1(4): 419-422

[14] Chen X, Wu S and Zhou J 2013 Influence of porosity on compressive and tensile strength of cement mortar. Constr. Build. Mater. 40(3): 869-874

[15] Popovics S 1985 New formulas for the prediction of the effect of porosity on concrete strength. ACI J. Proc. 82(2): 136146

[16] Shi C 1996 Strength, pore structure and permeability of alkali-activated slag mortars. Cem. Concr. Res. 26(10): 17891799

[17] O'Farrell M, Wild S and Sabir B B 2001 Pore size distribution and compressive strength of waste clay brick mortar. Cem. Concr. Res. 23(1): 81-91

[18] Wen C E, Yamada Y, Shimojima K, Chino Y, Hosokawa H and Mabuchi M 2004 Compressibility of porous magnesium foam: Dependency on porosity and pore size. Mater. Lett. 58(3-4): 357-360

[19] Pecqueux F, Tancret F, Payraudeau N and Bouler J M 2010 Influence of microporosity and macroporosity on the mechanical properties of biphasic calcium phosphate bioceramics: Modeling and experiment. J. Eur. Ceram. Soc. 30(4): 819829

[20] Tang L 1986 A study of the quantitative relationship between strength and pore-size distribution of porous materials. Cem. Concr. Res. 16(1): 87-96 
[21] Ryshkewitch E 1953 Compression strength of porous sintered alumina and zirconia. J. Am. Ceram. Soc. 36(2): 65-68

[22] Laskar M A I, Kumar R and Bhattacharjee B 1997 Some aspects of evaluation of concrete through mercury intrusion porosimetry. Cem. Concr. Res. 27(1): 93-105

[23] Shi D, Brown P W and Ma W 1991 Lognormal simulation of pore size distribution in cementitious materials. J. Am. Ceram. Soc. 74(6): 1861-1867

[24] Deo O and Neithalath N 2011 Compressive response of previous concretes proportioned for desired porosities. Constr. Build. Mater. 25(9): 4181-4189
[25] Todinov M T 2009 Is Weibull distribution the correct model for predicting probability of failure initiated by noninteracting flaws? Int. J. Solids Struct. 46(3-4): 887-901

[26] Savolainen P T, Mannering F L, Lord D and Quddus M A 2011 The statistical analysis of highway crash-injury severities: A review and assessment of methodological alternatives. Accid. Anal. Prev. 43(3): 1666-1676

[27] Poon C S, Kou S C and Lam L 2006 Compressive strength, chloride diffusivity and pore structure of high performance metakaolin and silica fume concrete. Constr. Build. Mater. 20(8): 858-865 